\title{
Genetic Basis and Clonal Population Structure of Antibiotic Resistance in Campylobacter jejuni Isolated From Broiler Carcasses in Belgium
}

\begin{abstract}
Mohamed Elhadidy ${ }^{1,2 *}$, William G. Miller ${ }^{3}$, Hector Arguello ${ }^{4}$, Avelino Álvarez-Ordóñez ${ }^{5}$, Alexandra Duarte ${ }^{6,7}$, Katelijne Dierick $^{7}$ and Nadine Botteldoorn ${ }^{7}$

${ }^{1}$ Department of Bacteriology, Mycology and Immunology, Faculty of Veterinary Medicine, Mansoura University, Mansoura, Egypt, ${ }^{2}$ University of Science and Technology, Zewail City of Science and Technology, Giza, Egypt, ${ }^{3}$ Produce Safety and Microbiology Research Unit, Agricultural Research Service, U.S. Department of Agriculture, Albany, CA, United States, ${ }^{4}$ Genomics and Animal Breeding, Department of Genetics, Faculty of Veterinary Science, University of Córdoba, Córdoba, Spain, ${ }^{5}$ Department of Food Hygiene and Technology, Institute of Food Science and Technology, University of León, León, Spain, ${ }^{6}$ Laboratory of Food Microbiology and Food Preservation, Department of Food Safety and Food Quality, Faculty of Bioscience Engineering, Ghent University, Gent, Belgium, ${ }^{7}$ National Reference Laboratory for Campylobacter, Scientific Institute of Public Health (WIV-ISP), Scientific Service: Foodborne Pathogens, Brussels, Belgium
\end{abstract}

Human campylobacteriosis is the leading food-borne zoonosis in industrialized countries.

OPEN ACCESS

Edited by:

Paula Teixeira,

Universidade Católica Portuguesa,

Portugal

Reviewed by:

Xu Jia,

Chengde Medical College, China Ben Pascoe,

University of Bath, United Kingdom

*Correspondence:

Mohamed Elhadidy

mm_elhadidy@mans.edu.eg

Specialty section:

This article was submitted to

Food Microbiology,

a section of the journal

Frontiers in Microbiology

Received: 03 March 2018

Accepted: 30 April 2018

Published: 17 May 2018

Citation:

Elhadidy M, Miller WG, Arguello $H$,

Álvarez-Ordóñez A, Duarte $A$, Dierick K and Botteldoorn N (2018) Genetic Basis and Clonal Population Structure of Antibiotic Resistance in Campylobacter jejuni Isolated From Broiler Carcasses in Belgium. Front. Microbiol. 9:1014. doi: 10.3389/fmicb.2018.01014
This study characterized the clonal population structure, antimicrobial resistance profiles and occurrence of antimicrobial resistance determinants of a set of Campylobacter jejuni strains isolated from broiler carcasses in Belgium. Minimum inhibitory concentrations (MICs) against five commonly-used antibiotics (ciprofloxacin, nalidixic acid, tetracycline, gentamicin, and erythromycin) were determined for 204 C. jejuni isolates. More than half of the isolates were resistant to ciprofloxacin or nalidixic acid. In contrast, a lower percentage of screened isolates were resistant to gentamicin or erythromycin. C. jejuni isolates resistant to ciprofloxacin and/or nalidixic acid were screened for the substitution T86l in the quinolone resistance determining region (QRDR) of the gyrA gene, while C. jejuni isolates resistant to tetracycline were screened for the presence of the tet(O) gene. These resistance determinants were observed in most but not all resistant isolates. Regarding resistance to erythromycin, different mutations occurred in diverse genetic loci, including mutations in the $23 S$ rRNA gene, the rp/D and rp/V ribosomal genes, and the intergenic region between $\mathrm{cme} R$ and $\mathrm{cme} A B C$. Interestingly, and contrary to previous reports, the $\mathrm{A} 2075 \mathrm{G}$ transition mutation in the $23 \mathrm{~S}$ rRNA gene was only found in one strain displaying a high level of resistance to erythromycin. Ultimately, molecular typing by multilocus sequence typing revealed that two sequence types (ST-824 and ST-2274) were associated to quinolones resistance by the presence of mutations in the gene gyrA $(p=0.01)$. In addition, ST-2274 was linked to the CIP-NAL-TET-AMR multidrug resistant phenotype. In contrast, clonal complex CC-45 was linked to increased susceptibility to the tested antibiotics. The results obtained in this study provide better understanding of the phenotypic and the molecular basis of antibiotic resistance in C. jejuni, unraveling some the mechanisms which confer antimicrobial resistance and particular clones associated to the carriage and spread of resistance genes.

Keywords: broiler, antibiotic resistance, resistance genes, mutations, MLST 


\section{INTRODUCTION}

Campylobacter spp., and in particular Campylobacter jejuni, are considered among one of the most prevalent zoonotic foodborne pathogens associated with sporadic diarrhea in humans. In the European Union, human campylobacteriosis is by far the most frequent foodborne infection, with more than 200,000 confirmed human cases/year (EFSA, 2015). Most of the cases are self-limiting and include symptoms such as fever, abdominal cramps and watery to bloody diarrhea. In severe cases, infection might lead to post-infectious neurological complications such as Guillain-Barré syndrome and MillerFisher syndrome (Humphrey et al., 2007). Consumption of contaminated poultry is the main source of human Campylobacter infections (Kaakoush et al., 2015). In poultry, the pathogen colonizes the gut in relatively high concentrations but produces few or no clinical symptoms (Luangtongkum et al., 2006). These high numbers of bacteria can contaminate broiler carcasses during slaughtering, with the subsequent transmission of Campylobacter through the food chain (Jeffrey et al., 2001).

Frequent isolation of antimicrobial-resistant Campylobacter strains of food animal origin represents a public health threat, as these sources may act as an important vehicle for the transmission of resistant strains to humans, where the spectrum of antibiotics efficient for disease treatment will be limited (Aarestrup and Engberg, 2001). As poultry and poultry products are considered the main reservoir for Campylobacter foodborne transmission, the intensive use of antimicrobial agents for therapy, prophylaxis, or as growth promoters in poultry production may lead to the selection of resistant strains that can be transmitted to humans via contaminated food (Aarestrup and Engberg, 2001). The use of antibiotics as growth promoters was banned in Europe in 2006 based on the European Community directive 90/167/EEC. Furthermore, in 2007, the World Health Organization (WHO) made recommendations for limiting the routine use of antimicrobials in production animals (Collignon et al., 2009). All EU member states are required to monitor antimicrobial resistance (AMR) in broiler flocks. The European Surveillance of Veterinary Antimicrobial Consumption (ESVAC) illustrates recent efforts of the European Medicines Agency to develop a central database on the use of antimicrobial agents in animals (EMA, 2009). Indeed, different antimicrobial consumption monitoring programs aimed at mitigating the risk related to antimicrobial resistance have been established in some European Countries, and Belgium is currently developing a veterinary antimicrobial consumption monitoring system (www. belvetsac.ugent.be).

Serious concerns associated with the increasing isolation frequency of resistant Campylobacter spp. have prompted the investigation of the resistance mechanisms to these antimicrobials. In Campylobacter, high levels of resistance to quinolones and fluoroquinolones are mainly due to single point mutations in the DNA gyrase gene gyrA, especially a C257T mutation that results in a T86I substitution (Iovine, 2013). The main mechanism that confers high-level resistance to macrolides in Campylobacter is the occurrence of point mutations in the peptidyl encoding region of the 23S rRNA gene (Vacher et al., 2005). In addition, modifications of the L4 or L22 ribosomal proteins, mediated by point mutations in the $r p l D$ and $r p l V$ genes, respectively, and the presence of the $23 \mathrm{~S}$ rRNA methyltransferase gene $\operatorname{erm} B$ have been also identified as resistance mechanisms for macrolides (Pérez-Boto et al., 2010; Wang et al., 2014; Florez-Cuadrado et al., 2017). Overexpression of the $C m e A B C$ multidrug efflux pump also works synergistically with these resistance mechanisms to confer resistance to the action of macrolides (Gibreel et al., 2005). Resistance to tetracycline, on the other hand, is mediated by the ribosomal protection protein TetO that is encoded by the tet $(\mathrm{O})$ gene, located on either the chromosome or on conjugatable plasmids (Gibreel et al., 2004; Wu et al., 2014).

MLST locus alleles have been previously used as a successful source-attribution model and in assigning genomes to host reservoir (Pascoe et al., 2017). In addition to the resistance mechanisms described above, several reports have previously highlighted the association of particular sequence types with certain antimicrobial resistance phenotypes in Europe, suggesting potential clonal expansion of antimicrobial resistance phenotypes in recent years (Habib et al., 2009; Cody et al., 2012; Kittl et al., 2013; Kovač et al., 2014, 2015; Klein-Jöbstl et al., 2016).

The aim of this study is to determine the frequency of antimicrobial resistance in a subset of 204 C. jejuni isolates recovered from broiler carcass swabs in Belgium, and to investigate the molecular mechanisms of resistance and the possible associations between antimicrobial resistance profiles and certain MLST genotypes.

\section{MATERIALS AND METHODS}

\section{Bacterial Strains and Growth Conditions}

A collection of 204 C. jejuni isolates were obtained between 2006 and 2015 from broiler carcass samples collected from different slaughter lines according to the ISO 10272-1 method (ISO 10272-1:ISO, 2006). All isolates were subcultured from $-80^{\circ} \mathrm{C}$ frozen stocks onto Columbia agar (Oxoid, United Kingdom) with 5\% horse blood (Sigma-Aldrich, United Kingdom). Plates were incubated at $41.5 \pm 1^{\circ} \mathrm{C}$ within a gas jar under microaerobic conditions $\left(6 \% \mathrm{O}_{2}, 7 \% \mathrm{CO}_{2}, 7 \% \mathrm{H}_{2}\right.$, and $\left.80 \% \mathrm{~N}_{2}\right)$, provided by the Anoxomat (Mark II System, The Netherlands).

\section{Phenotypic Antibiotic Resistance Profiling}

Testing for resistance to ciprofloxacin, erythromycin, gentamicin, nalidixic acid and tetracycline was carried out by the standard broth microdilution method, using the commercial diagnostic test for Campylobacter minimum inhibitory concentrations (MICs) (Sensititre ${ }^{\circledR}$ plates; Sensititre Campylobacter plate-EUCAMP, Trek Diagnostic Systems, UK) and following the manufacturer's instructions. Briefly, bacterial suspensions adjusted at approximately $1.5 \times 10^{8} \mathrm{CFU} / \mathrm{ml}$ (equivalent to $0.5 \mathrm{McF}$ arland standard) were inoculated into 96well microtiter plates containing Mueller-Hinton broth (Oxoid, UK) supplemented with 5\% defibrinated horse blood. Following bacterial inoculation, the Sensititre Campylobacter Plates were incubated under microaerobic conditions (Anoxomat, 
Mark II System, The Netherlands) at $37^{\circ} \mathrm{C}$ for $48 \mathrm{~h}$. The MIC was read as the lowest concentration completely inhibiting visible growth. Resistance against antibiotics was interpreted using the epidemiologic cut-off values (ECOFFs), following the guidelines of the European Committee on Antimicrobial Susceptibility Testing (EUCAST, 2015) (www.eucast.org). C. jejuni strain ATCC 33560 was included as a quality control in the antimicrobial susceptibility determinations.

\section{Molecular Characterization of Antimicrobial Resistance Phenotypes}

Resistance mechanisms were evaluated for quinolones and fluoroquinolones (nalidixic acid and ciprofloxacin, respectively), macrolides (erythromycin) and tetracycline. DNA was extracted from overnight bacterial cultures using a DNeasy Blood \& Tissue Kit (Qiagen, Germany) according to the manufacturer's instructions. DNA was eluted in $100 \mu \mathrm{l}$ of the kit elution buffer and stored at $-20^{\circ} \mathrm{C}$ for further molecular analysis of resistance determinants. C. jejuni isolates displaying resistance to ciprofloxacin and/or nalidixic acid were screened for a point mutation that results in the T86I substitution in the quinolone resistance determining region (QRDR) of gyrA, using the mismatch amplification mutation assay (MAMA-PCR) as previously described (Zirnstein et al., 1999). C. jejuni isolates resistant to tetracycline were screened for the presence of the $\operatorname{tet}(\mathrm{O})$ gene, as previously described by Gibreel et al. (2004). Erythromycin-resistant isolates were characterized for five genetic loci potentially responsible for resistance: the $23 \mathrm{~S}$ rRNA gene; the rplD and rplV 50S ribosomal subunit genes; the erm $B$ gene; and the intergenic region between $c m e R$ and $c m e A B C$. The mismatch amplification mutation assay previously described by Alonso et al. (2005) was used to identify A2074C and A2075G point mutations in the $23 \mathrm{~S}$ rRNA gene previously associated with high-level erythromycin resistance. Modifications in the L4 and L22 ribosomal proteins were determined using amplification and sequencing of the $r p l D$ and $r p l V$ genes, respectively, as reported elsewhere (Corcoran et al., 2006). The presence of the $\mathrm{ermB}$ gene among resistant strains was assessed previously described by Zhou et al. (2016). Finally, different polymorphisms in the regulatory region of $c m e A B C$ were screened by PCR amplification and sequencing of the intergenic region between the $c m e R$ and $c m e A$ genes. All protocols, primer sequences, amplification and sequencing conditions were employed as described by referenced authors.

\section{DNA Sequence Analysis}

Amplification products generated were purified using the QIAquick PCR Purification kit (Qiagen, Germany). Purified amplicons were sequenced using the ABI PRISM BigDye terminator cycle sequencing kit (ver. 3.1; Life Technologies, Grand Island, NY) and standard protocols. DNA sequencing was performed on an ABI PRISM 3730 DNA Analyzer (Life Technologies), using POP-7 polymer and ABI PRISM Genetic Analyzer Data Collection and ABI PRISM Genetic Analyzer Sequencing Analysis software. Sequences were manually edited and then compared to those in the current databases using the BLAST suite of programs. Sequence alignments and SNP identification was performed using the Lasergene analysis package (v. 8.0; DNASTAR, Madison, WI).

\section{Analysis of Genetic Similarity Among Antimicrobial-Resistant Strains}

The genetic similarity of antimicrobial-resistant isolates was analyzed using multilocus sequence typing (MLST) as previously described (Miller et al., 2005). Amplicons were sequenced as described above. Allele numbers, sequence types (STs) and clonal complexes (CCs) were assigned by submitting the DNA sequences to the Campylobacter PubMLST database website (https://pubmlst.org/campylobacter/) at the University of Oxford.

\section{Statistical Analysis}

Descriptive statistical analysis of the data was performed using $\mathrm{R}$ version 3.3.2 (R-project). Association among the different antimicrobial resistance determinants, and between AMR profiles and MLST STs and CCs were examined using Chi2 or Fisher's exact tests. The same statistical tests were used to establish changes or trends in susceptibility to the antimicrobials tested. Significance was established at $\alpha=0.05$.

\section{RESULTS}

\section{Antimicrobial Resistance Phenotypes}

Fifty-eight (28.4\%) isolates were pan-susceptible to all antimicrobials tested. The highest frequency of resistance was observed for ciprofloxacin (53.9\%), followed by resistance to nalidixic acid (53.4\%), and tetracycline (47\%) (Table 1). In this study, almost all (95.5\%) ciprofloxacin resistant isolates were cross-resistant to nalidixic acid. A low frequency of resistance was observed for gentamicin (6.9\%) and erythromycin (4.9\%). MIC tests yielded 18 different antimicrobial resistance patterns (Table 2).

\section{Genotypic Characterization of Antimicrobial Resistance}

Isolates that were resistant to ciprofloxacin and/or nalidixic acid were screened for T86I mutations in the gyrA gene. A total of 98 isolates that were simultaneously resistant to Cip and Nal harbored a C257T point mutation that resulted in a T86I substitution. Five isolates that showed simultaneous resistance to both antibiotics were negative for this mutation. Four $\mathrm{Cip}^{\mathrm{r}}$

TABLE 1 | Antimicrobial resistance rates of $C$. jejuni from broiler carcasses.

\begin{tabular}{lllcc}
\hline Rank Class & Antimicrobial & $\begin{array}{c}\text { Break points } \\
\text { (mg/L) }\end{array}$ & $\begin{array}{c}\text { No. of resistant } \\
\text { isolates (\%) }\end{array}$ \\
\hline Aminoglycosides & Gentamicin & $>2$ & $14(6.9 \%)$ \\
Macrolides & Erythromycin & $>4$ & $10(4.9 \%)$ \\
Quinolones and & Ciprofloxacin & $>0.5$ & $110(53.9 \%)$ \\
fluoroquinolones & & & \\
& & Nalidixic acid & $>16$ & $109(53.4 \%)$ \\
$\|$ & Tetracyclines & Tetracycline & $>1$ & $96(47 \%)$
\end{tabular}


TABLE 2 | Distribution of antimicrobial resistance patterns and MLST sequence types among C. jejuni from broiler carcasses.

\begin{tabular}{|c|c|c|}
\hline Antimicrobial resistance profile ${ }^{a}$ & No. of isolates $n(\%)$ & Sequence types \\
\hline \multirow[t]{4}{*}{ Sensitive } & $58(28.4 \%)$ & ST-19 (1), ST-137 (2), ST-21 (4), ST-25 (1), ST-38 (1), \\
\hline & & $\begin{array}{l}\text { ST-42 (1), ST-45 (7), ST-48 (2), ST-50 (7), ST-51 (1), ST-61 (1), ST-257 (2), ST-267 (1), ST-400 } \\
\text { (1), ST-415 (1), ST-418 (1), }\end{array}$ \\
\hline & & ST-475 (1), ST-464 (1), ST-538 (1), ST-583 (3), ST-606 (1), \\
\hline & & ST-607 (1), ST-791 (1), ST-1044 (4), ST-1045 (1), ST-1326 (1), \\
\hline \multirow[t]{2}{*}{ TET } & $25(12.2 \%)$ & $\begin{array}{l}\text { ST-48 (1), ST-50 (1),ST-141 (1), ST-257 (1), ST-464 (1), ST-879 (4), ST-2314 (1),ST-2324 (1), } \\
\text { ST-2547 (1), ST-2641 (1), ST-2803 (1), ST-2844 (2), ST-3547 (1) }\end{array}$ \\
\hline & & $\begin{array}{l}\text { ST-4602 (1), ST-5970 (1), ST-7953 (1), ST-7954 (1), ST-7960 (1), ST-8636 (1), ST-8637 (1), } \\
\text { ST-8639 (1) }\end{array}$ \\
\hline CIP & $3(1.5 \%)$ & ST-572 (1), ST-775 (1), ST-2132 (1) \\
\hline & & ST-122 (1), ST-267 (1), ST-305 (1), ST-324 (1), ST-572 (2), \\
\hline & & ST-607 (1), ST-775 (1), ST-824 (4), ST-883 (1), ST-982 (1), \\
\hline & & $\begin{array}{l}\text { ST-905 (1), ST-1519 (1), ST-1707 (1), ST-2258 (1), ST-4800 (1), ST-5018 (2), ST-8638 (1), } \\
\text { ST-8640 (1) }\end{array}$ \\
\hline NAL TET & $2(1 \%)$ & ST-51 (1), ST-2844 (1) \\
\hline CIP TET & $1(0.5 \%)$ & ST-2882 (1) \\
\hline ERY TET & $1(0.5 \%)$ & ST-1519 (1) \\
\hline \multirow[t]{3}{*}{ Q TET } & $58(28.4 \%)$ & ST-19 (2), ST-21 (2), ST-44 (1), ST-46 (2), ST-48 (1), ST-50 (3), \\
\hline & & ST-51 (1), ST-141(1), ST-354 (2), ST-492 (1), ST-464 (5), \\
\hline & & ST-824 (1), ST-877 (1), ST-883 (2), ST-904 (2), ST-969 (1), \\
\hline Q GEN TET & $1(0.5 \%)$ & ST-606 (1) \\
\hline Q ERY GEN & $1(0.5 \%)$ & ST-50 (1) \\
\hline Q ERY TET & $1(0.5 \%)$ & ST-262 (1) \\
\hline Q ERY GEN TET & $4(2 \%)$ & ST-48 (1), ST-354 (1), ST-824 (1), ST-8641 (1) \\
\hline
\end{tabular}

${ }^{a} M D R$ strains are in bold and underlined. Q: CIP+NAL.

$\mathrm{Nal}^{\mathrm{s}}$ isolates presented C257T point mutations in the QRDR. However, two $\mathrm{Cip}^{\mathrm{r}} \mathrm{Nal}^{\mathrm{s}}$ isolates (cj1396, cj2940) had no C257T point mutation in the gyrA gene; similarly, none of the six Cip ${ }^{\mathrm{s}}$ $\mathrm{Nal}^{\mathrm{r}}$ isolates harbored this mutation in the gyrA gene. Isolates that were resistant to ciprofloxacin and/or nalidixic acid but had no C257T mutation in the gyrA gene showed MICs in the range of 4-32 mg/L for CIP and 32-256 mg/L for Nal.

The erythromycin-resistant strains were screened for the presence of $23 \mathrm{~S}$ rRNA, rplD, rplV and cmeRABC locus mutations tentatively associated previously with erythromycin resistance in Campylobacter. Within the 23S rRNA gene of these strains, the $\mathrm{A} 2074 \mathrm{G}$ mutation was not observed in any of the isolates and the A2075G mutation was identified in only one isolate displaying a high level of erythromycin resistance (>256 mg/L).
Further analysis of the rplD and rplV genes (encoding the 50S ribosomal subunit proteins L4 and L22, respectively) identified several different potential amino acid substitutions (Table 3). The following predicted amino acid substitutions were observed in L4: V121A (two isolates), T177S (one isolate), M192I (two isolates), V196A (five isolates). For L22, more diverse amino acid substitutions were identified including: Q24R (one isolate), V65I (eight isolates), G74A (ten isolates), A103V (one isolate), T109S (ten isolates), A111E (ten isolates), and A114T (ten isolates). Using the erythromycin-sensitive C. jejuni strain NCTC 11168 as a "wild-type" reference strain, four CmeR alleles were identified among the erythromycin-resistant isolates (Table 3, Figure S1A). Additionally, sequencing of the cme RAIVS (cmeRcmeA intervening sequence) region identified three alleles, with 


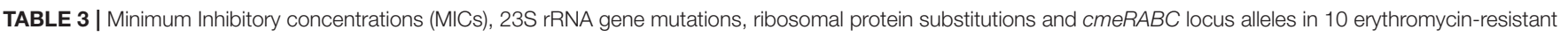
C. jejuni isolates.

\begin{tabular}{|c|c|c|c|c|c|c|c|}
\hline \multirow[t]{2}{*}{ Strain } & \multirow[t]{2}{*}{ Ery MIC (mg/L) } & \multirow{2}{*}{$\begin{array}{l}\text { Mutation in 23S } \\
\text { rRNA gene }\end{array}$} & \multirow[t]{2}{*}{ ermB } & \multicolumn{2}{|c|}{ Ribosomal protein polymorphisms } & \multirow[t]{2}{*}{ cmeR } & \multirow[t]{2}{*}{ RAIVS } \\
\hline & & & & L4 mutation & L22 mutation & & \\
\hline 810 & $>256$ & A2075G & - & A196V & V65I, G74A, T109S, A111E, A114T & 3 & 3 \\
\hline 1062 & $>256$ & WT & - & A196V & V65I, G74A, T109S, A111E, A114T & WT & WT \\
\hline 1396 & $>256$ & WT & - & 0 & G74A, A103V, T109A, A111E, A114T & 4 & 4 \\
\hline 656 & 12 & WT & - & 0 & Q24R, V65I, G74A, T109A, A111E, A114T & 2 & 2 \\
\hline 713 & 128 & WT & - & A196V & V65I, G74A, T109S, A111E, A114T & NT & NT \\
\hline 783 & 32 & WT & - & A196V & V65I, G74A, T109S, A111E, A114T & 3 & 3 \\
\hline 856 & 6 & WT & - & M192I, V121A & G74A, T109A, A111E, A114T & 2 & 2 \\
\hline 872 & $>256$ & WT & - & 0 & V65I, G74A, T109S, A111E, A114T & WT & WT \\
\hline 1680 & $>256$ & WT & - & M192I, V121A, T177S & V65I, G74A, T109A A111E, A114T & 2 & 2 \\
\hline 2136 & 12 & WT & - & A196V & V65I, G74A, T109S, A111E, A114T & 5 & 4 \\
\hline
\end{tabular}

WT, wild type (with respect to C. jejuni strain NCTC 11168); NT, not tested; RAIVS, cmeRA intervening sequence. For cme locus alleles, see also Figure S1.

respect to strain NCTC 11168 (Table 3, Figure S1B). In addition to two SNPs, allele cmeRAIVS4 contained a six base insertion.

\section{Association of MLST Sequence Types (STs) and Clonal Complexes (CCs) With Antimicrobial Resistance Patterns and gyrA Mutations}

The analysis was restricted only on those STs and CCs represented by five or more isolates. The distribution of MICs for the different antibiotics among the sequence types revealed minor differences, except for strains from ST-824 $(p=0.016)$ and ST-2274 $(p=0.002)$, which were significantly more resistant to ciprofloxacin and nalidixic acid than strains from other STs (Figure 1). The distribution of MICs for the different antibiotics among the clonal complexes revealed that strains from CC45 were more sensitive against ciprofloxacin $(p<0.001)$, tetracycline $(p=0.0201)$ and nalidixic acid $(p=0.047)$ than strains from other clonal complexes.

The statistical analyses revealed that three STs were significantly associated with the presence of gyrA mutations. These are ST-2274 $(p=0.001)$, and ST-824 $(p=0.02)$, which showed a higher proportion of isolates with gyrA mutations. On the other hand, ST-45 did not show any isolate with gyrA mutation $(p=0.01)$. The prevalence of gyrA mutations was also significantly linked to the clonal complex CC-574 ( $p=0.02937)$, while all CC-45 ( $p=0.047)$ isolates showed no gyrA mutations.

\section{DISCUSSION}

The rising trend of antimicrobial resistance among C. jejuni strains represents a serious public health concern. In recent years, many studies conducted worldwide reported high levels of resistance to ciprofloxacin and tetracycline, and emerging resistance to macrolides (Engberg et al., 2001; Luangtongkum et al., 2009). Therefore, continuous monitoring of resistance rates and mechanisms of resistance is crucial to combat the potential spread of AMR C. jejuni across the food chain. This study was conducted to provide better insight into the dynamics and molecular epidemiology of C. jejuni antibiotic resistance by characterizing 204 isolates from broiler carcasses obtained in Belgium over a decade (2006-2015). The majority of antimicrobials screened (all antimicrobials used except tetracycline) are critically important for human health, being classified in the Rank I by the World Health Organization (WHO, 2011).

In this study, approximately half of the isolates were resistant to nalidixic acid (53.9\%), ciprofloxacin (53.4\%) or tetracycline (47\%). These levels of resistance are consistent with recent data obtained in another Belgian study carried out in 2007 that analyzed C. jejuni isolates from chicken meat (Habib et al., 2009). However, these resistance levels are moderately higher than those reported in other studies previously conducted in Belgium (Van Looveren et al., 2001; Mattheus et al., 2012). Mattheus et al. (2012) performed a surveillance analysis in poultry meat over the period 2004-2009, reporting the following resistance figures: $39.5 \%$ for nalidixic acid, $38.0 \%$ for ciprofloxacin and $40.8 \%$ for tetracycline. Similar findings were observed in 1998 in another study of AMR that included C. jejuni strains isolated from broilers, where rates of resistance to nalidixic acid, ciprofloxacin and tetracycline were $44.2,44.2$, and $34.4 \%$, respectively (Van Looveren et al., 2001). The combination of these results reflects the relatively high resistance to these antimicrobials among Belgian C. jejuni isolates and a slight increase of AMR in C. jejuni over the last two decades in poultry production. Fluoroquinolones have commonly been used to treat infection in poultry production, while tetracyclines have been frequently used in animal production to treat infections and as growth promoters over the last 50 years (Giacomelli et al., 2014). The high resistance rates observed for these antimicrobials may be the consequence of their continuous overuse.

C. jejuni isolates that were resistant to ciprofloxacin and/or nalidixic acid were screened for the presence of a T86I amino acid substitution in the QRDR of the gyrA gene. The present results 


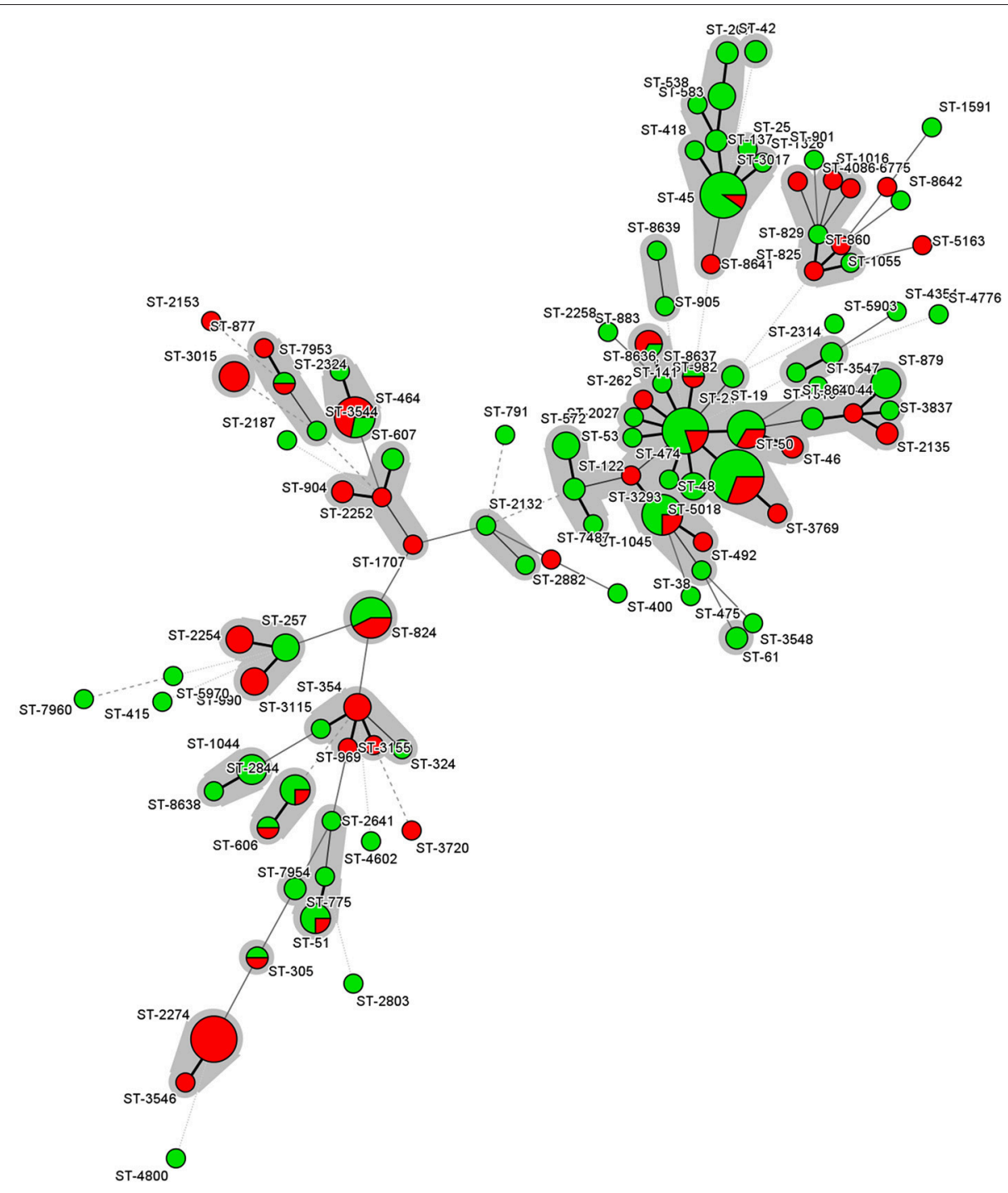

FIGURE 1 | Minimum spanning tree of MLST data for the 204 C. jejuni isolates. Each node represents a particular ST, and the size of the circle is proportional to the number of isolates sharing the same ST. Inside the circles, the colors reflect the number of antimicrobial resistances. Green color represents isolates with less than 3 antimicrobial resistances while red color represents isolates with three or more resistances. Each individual sequence type is distinguished by separate circles and linked by lines indicating allelic variation. STs belonging to the same CC are color-shaded.

showed that this mutation was absent in some isolates that were resistant only to ciprofloxacin or nalidixic acid, and five isolates that showed simultaneous resistance to both antimicrobials. This observation is in agreement with previous reports that suggest that this substitution does not confer universal resistance to all quinolone antibiotics and concluded that quinolone resistance might also be attributed to other unknown resistance mechanisms (Dionisi et al., 2004; Corcoran et al., 2005; Bolton et al., 2013). This idea is further supported by the fact that although T86I mutations in the gyrA gene have been previously reported to be associated with high levels of resistance to nalidixic acid (MIC 64-256 mg/L) and ciprofloxacin (MIC 16-64 mg/L) (Engberg et al., 2001), we identified three $\mathrm{Nal}^{\mathrm{r}}$ isolates (cj473, cj3408, cj2500), one $\mathrm{Cip}^{\mathrm{r}}$ isolate (cj2940) and two $\mathrm{Nal}^{\mathrm{r}} \mathrm{Cip}^{\mathrm{r}}$ isolates (cj1062, cj857) without the gyrA T86I mutation that nevertheless exhibited MICs within those ranges. Future studies will be directed to identify additional mutations, other than gyrA T86I, that also confer resistance to ciprofloxacin and/or nalidixic acid.

In this study, the low resistance rates to erythromycin and gentamicin are consistent with earlier reports in Belgian $C$. jejuni strains isolated from broiler meat. For instance, previously reported resistance levels to erythromycin and gentamicin were as low as 0.7 and 1.4\% (Habib et al., 2009), 6.3 and 0\% (Van Looveren et al., 2001), and 6 and 12.9\% (Mattheus et al., 2012). These results are also in agreement with another European study performed in Italy (Giacomelli et al., 2014). Despite the common use of macrolides in poultry, for instance the use of tylosin in young birds (Giacomelli et al., 2014), the low resistance to erythromycin found in our study may be due to the 
slower development of erythromycin-resistant mutants during exposure to antibiotics, the reduced biological fitness of resistant mutants and/or their reduced ability to survive in the absence of selective pressure (Luangtongkum et al., 2009; Luangtonkum et al., 2012). The infrequent isolation of strains resistant to the aminoglycoside gentamicin might be attributed to the rare use of this antimicrobial agent as a prophylactic or therapeutic agent in broiler production systems (Rodrigo et al., 2007).

A2074G and A2075G mutations in region V of the 23S rRNA gene have been previously reported as prominent contributors to high-level macrolide resistance in Campylobacter (Niwa et al., 2001; Vester and Douthwaite, 2001; Haanperä et al., 2005; Corcoran et al., 2006; Caldwell et al., 2008). However, in this study, A2074G was not identified and A2075G was identified in only one isolate displaying high level erythromycin resistance. Indeed, the A2075G mutation was absent in five highlevel erythromycin-resistant isolates (MIC > $128 \mathrm{mg} / \mathrm{L}$ ). These findings are similar to previous observations from broiler flocks in Italy, where only $3.1 \%(1 / 36)$ of the macrolide-resistant $C$. jejuni isolates carried the A2075G mutation in the $23 \mathrm{~S}$ rRNA gene and none harbored the A2074G mutation (Giacomelli et al., 2012). This observation could be due to a lower frequency of occurrence of these mutations in the $23 \mathrm{~S}$ rRNA gene in $C$. jejuni when compared to $C$. coli as previously hypothesized by Giacomelli et al. (2012). The absence of this mutation among low-level erythromycin-resistant isolates was nonetheless not unexpected, as this mutation has been linked to high-level erythromycin resistance (Alonso et al., 2005). These results prompted us to investigate other possible alternative mechanisms of macrolide resistance, including modifications in the $50 \mathrm{~S}$ ribosomal subunit proteins L4 and L22 and modifications at the CmeABC efflux pump locus.

As ribosomal components, the $50 \mathrm{~S}$ subunit proteins L4 and L22 are highly conserved proteins in bacteria. Therefore, mutations in the genes encoding the L4 and L22 proteins affect the binding of macrolides to the $50 \mathrm{~S}$ ribosomal subunit, resulting in macrolide resistance (Gibreel and Taylor, 2006; Belanger and Shryock, 2007). In Campylobacter, mutations in the large loop of the L4 protein (residues 55-77) and the L22 protein (residues 78-98) have been confirmed to be associated with macrolide resistance in various strains. In the present study, all mutations observed were found outside of the loop regions of the L4 or L22 proteins (Table 3). Furthermore, some of the identified mutations were previously identified both in resistant and susceptible isolates, for example V121A, A196V, A111E, and A114T in the L4 or L22 proteins (Corcoran et al., 2006). Thus, although it is possible that the ribosomal protein mutations observed here may contribute to macrolide resistance, it is likely that mutations external to the L4 and L22 proteins are the basis of the observed erythromycin resistance in these strains.

In $C$. jejuni, transcriptional regulation of the efflux pump operon $c m e A B C$ is carried out by the repressor $C m e R$, which binds specifically to an inverted repeat (IR) in the intervening sequence between $c m e R$ and $c m e A$ (Lin et al., 2005a). This IR overlaps the predicted -35 region in the $c m e A B C$ promoter (Lin et al., 2005b; see also Figure S1B). Lin et al. (2005b) demonstrated that a single bp deletion in the two deletion in the two bp IR spacer resulted in decreased CmeR binding with a concomitant elevation of fluoroquinolone resistance. In this study, mutations were identified in the $c m e R-c m e A$ intergenic region; however one of the mutations, A58T in alleles cmeRAIVS2-3 (Figure S1B), might be expected to improve the IR and thus lead to enhanced CmeR binding. Only one of the mutations, a six bp insertion in allele cmeRAIVS4, is predicted to disrupt the IR, increasing the spacer between the two IR half sites from two to eight bp. However, this insertion would also increase the spacing between the predicted -35 and $-16 /-10$ regions of $\mathrm{P}_{\mathrm{cmeABC}}$ and thus might result in decreased transcription of cmeABC, despite the potential loss of repression by $C m e R$. Mutations were also observed in CmeR (Table 3, Figure S1A); however, the effect of these mutations on macrolide resistance remains to be determined, especially as it is unknown how these CmeR alleles interact with the RAIVS IR region. Taken together with the results of the 23S rRNA and L4/L22 analyses, the genetic basis of macrolide resistance in the ten strains identified in this study is largely unknown. Additional research will be necessary, potentially requiring whole genome sequencing and further analysis of $C m e R$ binding to $\mathrm{P}_{\mathrm{cmeABC}}$, in order to determine the cause or causes of macrolide resistance in these strains.

Previous studies have observed an association between some sequence types (STs) or clonal complexes (CCs), in C. jejuni isolated from different sources, and resistance to quinolones and tetracycline, suggesting a local clonal expansion of resistance phenotypes arising from the use of antimicrobials in different animal and human settings (Habib et al., 2009; Cody et al., 2012; Kittl et al., 2013; Kovač et al., 2014, 2015; Klein-Jöbstl et al., 2016). The correlation between CC-45 and susceptibility to quinolones and tetracycline observed in this study is in agreement with a study conducted by Habib et al. (2009) on C. jejuni isolates from chicken meat preparations in Belgium. To the best of our knowledge, this is the first study that reports an association of ST-824 and ST-2274 with resistance to ciprofloxacin and nalidixic acid. Interestingly, the correlation between ST-2274 and the CIPNAL-TET AMR profile was notable. Considering that ST-2274 is among the most common STs found in Belgium (Elhadidy et al., 2018), these results suggest that ST-2274 is among the genotypes mainly responsible for the spread of this AMR pattern.

In conclusion, this study examined the mechanisms and the clonal expansion of antimicrobial resistance among $C$. jejuni strains isolated from broiler carcasses in Belgium. The previously-reported resistance mechanisms monitored in this study were not found in all resistant isolates, suggesting that resistance to the tested antibiotics might be attributed to other unknown resistance mechanisms.

\section{AUTHOR CONTRIBUTIONS}

ME conceived and designed the study, performed the tests, analyzed and interpreted the data, and wrote the manuscript. $\mathrm{WM}$ performed all sequencing experiments. WM, $\mathrm{AD}$, $\mathrm{KD}$, and $\mathrm{NB}$ substantially contributed in analysis of the results, and revision of the manuscript. AÁ-O, and HA conducted the data analysis and contributed to the writing 
of the manuscript. All authors read and approved the final manuscript.

\section{ACKNOWLEDGMENTS}

We would like to thank the Belgian Federal Science Policy (BELSBO) for supporting the post-doctoral fellowship of ME at WIV-ISP. The authors would like to thank Dr. Shannon Manning at the University of Michigan and Dr. Dorota Korsak at the University of Warsaw for providing positive controls for mutations in the 23S rRNA gene. Parts of this work were presented at the Twenty Seventh European conference of Clinical Microbiology and Infectious Diseases (ECCMID), April 21-25 2017, Vienna, Austria and the Twenty first Conference on Food Microbiology in Brussels, Belgium, 15-16 September 2016. AA-O acknowledges the financial support by Fundación BBVA and Ministerio de Economía y Competitividad (AGL201678085-P). HA is a post-doctoral researcher supported by the Juan de la Cierva Post-doctoral Trainee Program of the Spanish Ministry of Economy and Competitiveness (FJCI-201422877).

\section{SUPPLEMENTARY MATERIAL}

The Supplementary Material for this article can be found online at: https://www.frontiersin.org/articles/10.3389/fmicb. 2018.01014/full\#supplementary-material

Figure S1 Allelic variation within $C m e R$ and the $c m e R-c m e A$ intervening sequence. For the erythromycin-resistant isolates, the predicted $\mathrm{Cme} R$ protein sequences and the RAIVS (cmeR-cmeA intervening sequence) nucleotide sequences were determined. Relative to the erythromycin-sensitive $C$. jejuni strain NCTC 11168, four CmeR and three RAIVS alleles were identified. These alleles (and the respective sequences from strain NCTC 11168) were aligned using MegAlign (v. 8.0, DNASTAR, Madison, WI). Using the sequences of strain NCTC 11168 as "wild-type," amino acid substitutions within the CmeR alleles (A) and nucleotide changes within the RAIVS alleles (B) are boxed. Half sites of the inverted repeat (IR) are shaded in gray. Predicted-35, -16 , and -10 regions are labeled. Promoter and IR regions are as described in Lin et al. (2005a).

\section{REFERENCES}

Aarestrup, F. M., and Engberg, J. (2001). Antimicrobial resistance of thermophilic Campylobacter. Vet. Res. 32, 311-321. doi: 10.1051/vetres:2001127

Alonso, R., Mateo, E., Churruca, E., Martinez, I., Girbau, C., and FernándezAstorga, A. (2005). MAMA-PCR assay for the detection of point mutations associated with high-level erythromycin resistance in Campylobacter jejuni and Campylobacter coli strains. J. Microbiol. Methods. 63, 99-103. doi: 10.1016/j.mimet.2005.03.013

Belanger, A. E., and Shryock, T. R. (2007). Macrolide-resistant Campylobacter: the meat of the matter. J. Antimicrob. Chemother. 60, 715-723. doi: $10.1093 / \mathrm{jac} / \mathrm{dkm} 300$

Bolton, D., Patriarchi, A., Fox, Á., and Fanning, S. (2013). A study of the molecular basis of quinolone and macrolide resistance in a selection of Campylobacter isolates from intensive poultry flocks. Food Control. 30, 222-226. doi: 10.1016/j.foodcont.2012.06.044

Caldwell, D. B., Wang, Y., and Lin, J. (2008). Development, stability, and molecular mechanisms of macrolide resistance in Campylobacter jejuni. Antimicrob. Agents Chemother. 52, 3947-3954. doi: 10.1128/AAC.00450-08

Cody, A. J., McCarthy, N. M., Wimalarathna, H. L., Colles, F. M., Clark, L., Bowler, I. C., et al. (2012). A longitudinal 6-year study of the molecular epidemiology of clinical Campylobacter isolates in Oxfordshire, United Kingdom. J. Clin. Microbiol. 50, 3193-3201. doi: 10.1128/JCM.01086-12

Collignon, P., Powers, J. H., Chiller, T. M., Aidara-Kane, A., and Aarestrup, F. M. (2009). World Health Organization ranking of antimicrobials according to their importance in human medicine: a critical step for developing risk management strategies for the use of antimicrobials in food production animals. Clin. Infect. Dis. 49, 132-141. doi: 10.1086/599374

Corcoran, D., Quinn, T., Cotter, L., and Fanning, S. (2005). Relative contribution of target gene mutation and efflux to varying quinolone resistance in Irish Campylobacter isolates. FEMS Microbiol. Lett. 253, 39-46. doi: 10.1016/j.femsle.2005.09.019

Corcoran, D., Quinn, T., Cotter, L., and Fanning, S. (2006). An investigation of the molecular mechanisms contributing to high-level erythromycin resistance in Campylobacter. Int. J. Antimicrob. Agents. 27, 40-45. doi: 10.1016/j.ijantimicag.2005.08.019

Dionisi, A. M., Luzzi, I., and Carattoli, A. (2004). Identification of ciprofloxacinresistant Campylobacter jejuni and analysis of the gyrA gene by the Light Cycler mutation assay. Mol. Cell Probes 18, 255-261. doi: 10.1016/j.mcp.2004.02.001

EFSA, ECDC (2015). The european union summary report on trends and sources of zoonoses, zoonotic agents and food-borne outbreaks in 2014. EFSA J. 13:4329. doi: 10.2903/j.efsa.2015.4329

Elhadidy, M., Arguello, H., Álvarez-Ordóñez, A., Miller, W. G., Duarte, A., Martiny, D., et al. (2018). Orthogonal typing methods identify genetic diversity among Belgian Campylobacter jejuni strains isolated over a decade from poultry and cases of sporadic human illness. Int. J. Food Microbiol. 275, 66-75. doi: 10.1016/j.ijfoodmicro.2018.04.004

EMA European Medicines Agency (2009). European Surveillance of Veterinary Antimicrobial Consumption (ESVAC): Meeting Report. 2009. Available online at: www.ema.europa.eu (Accessed February 1, 2012).

Engberg, J., Aarestrup, F. M., Taylor, D. E., Gerner-Smidt, P., and Nachamkin, I. (2001). Quinolone and macrolide resistance in Campylobacter jejuni and C. coli: resistance mechanisms and trends in human isolates. Emerg. Infect. Dis. 7, 24-34. doi: 10.3201/eid0701.010104

EUCAST (2015). European Committee on Antimicrobial Susceptibility Testing Breakpoint Tables for Interpretation of MICs and Zone Diameters. Available online at: http://www.eucast.org/fileadmin/src/media/PDFs/EUC

Florez-Cuadrado, D., Ugarte-Ruiz, M., Meric, G., Quesada, A., Porrero, M. C., Pascoe, B., et al. (2017). Genome comparison of erythromycin resistant Campylobacter from turkeys identifies hosts and pathways for horizontal spread of erm(B) genes. Front. Microbiol. 8:2240. doi: $10.3389 /$ fmicb. 2017.02240

Giacomelli, M., Andrighetto, C., Rossi, F., Lombardi, A., Rizzotti, L., Martini, M., et al. (2012). Molecular characterization and genotypic antimicrobial resistance analysis of Campylobacter jejuni and Campylobacter coli isolated from broiler flocks in northern Italy. Avian Pathol. 41, 579-588. doi: 10.1080/03079457.2012.734915

Giacomelli, M., Salata, C., Martini, M., Montesissa, C., and Piccirillo, A. (2014). Antimicrobial resistance of Campylobacter jejuni and Campylobacter coli from poultry in Italy. Microb. Drug Resist. 20, 181-188. doi: 10.1089/mdr.2013.0110

Gibreel, A., and Taylor, D. E. (2006). Macrolide resistance in Campylobacter jejuni and Campylobacter coli. J. Antimicrob. Chemother. 58, 243-255. doi: $10.1093 / \mathrm{jac} / \mathrm{dkl} 210$

Gibreel, A., Kos, V. N., Keelan, M., Trieber, C. A., Levesque, S., Michaud, S., et al. (2005). Macrolide resistance in Campylobacter jejuni and Campylobacter coli: molecular mechanism and stability of the resistance phenotype. Antimicrob. Agents Chemother. 4, 2753-2759. doi: 10.1128/AAC.49.7.2753-2759.2005

Gibreel, A., Tracz, D. M., Nonaka, L., Ngo, T. M., Connell, S. R., and Taylor, D. E. (2004). Incidence of antibiotic resistance in Campylobacter jejuni isolated in Alberta, Canada, from 1999 to 2002, with special reference to tet (O)mediated tetracycline resistance. J. Antimicrob. Chemother. 48, 3442-3450. doi: 10.1128/AAC.48.9.3442-3450.2004

Haanperä, M., Huovinen, P., and Jalava, J. (2005). Detection and quantification of macrolide resistance mutations at positions 2058 and 2059 of the $23 \mathrm{~S}$ 
rRNA gene by pyrosequencing. Antimicrob. Agents Chemother. 49, 457-460. doi: 10.1128/AAC.49.1.457-460.2005

Habib, I., Miller, W. G., Uyttendaele, M., Houf, K., and De Zutter, L. (2009). Clonal population structure and antimicrobial resistance of Campylobacter jejuni in chicken meat from Belgium. Appl. Environ. Microbiol. 75, 4264-4272. doi: 10.1128/AEM.00168-09

Humphrey, T., O’Brien, S., and Madsen, M. (2007). Campylobacters as zoonotic pathogens: a food production perspective. Int. J. Food Microbiol. 117, 237-257. doi: 10.1016/j.ijfoodmicro.2007.01.006

Iovine, N. M. (2013). Resistance mechanisms in Campylobacter jejuni. Virulence 4, 230-240. doi: 10.4161/viru.23753

ISO - International Organization for Standardization (2006). ISO 10272 Microbiology of Food and Animal Feeding Stuff - Horizontal Method for Detection and Enumeration of Campylobacter spp. - Part 1: Enrichment Method; part 2: Enumeration Method. Geneva: ISO.

Jeffrey, J. S., Tonooka, K. H., and Lozanot, J. (2001). Prevalence of campylobacter spp. from skin, crop, and intestine of commercial broiler chicken carcasses at processing. Poul. Sci. 80, 1390-1392. doi: 10.1093/ps/80.9.1390

Kaakoush, N. O., Castaño-Rodríguez, N., Mitchell, H. M., and Man, S. M. (2015). Global epidemiology of Campylobacter infection. Clin. Microbiol. Rev. 28, 687-720. doi: 10.1128/CMR.00006-15

Kittl, S., Heckel, G., Korczak, B. M., and Kuhnert, P. (2013). Source attribution of human Campylobacter isolates by MLST and fla-typing and association of genotypes with quinolone resistance. PLOS ONE 14:e81796. doi: 10.1371/journal.pone.0081796

Klein-Jöbstl, D., Sofka, D., Iwersen, M., Drillich, M., and Hilbert, F. (2016). Multilocus sequence typing and antimicrobial resistance of Campylobacter jejuni isolated from dairy calves in Austria. Front. Microbiol. 7:72. doi: $10.3389 /$ fmicb.2016.00072

Kovač, J., Cadež, N., Lušicky, M., Nielsen, E. M., Ocepek, M., Raspor, P., et al. (2014). The evidence for clonal spreading of quinolone resistance with a particular clonal complex of Campylobacter jejuni. Epidemiol. Infect. 42, 2595-2603. doi: 10.1017/S0950268813003245

Kovač, J., Cadež, N., Stessl, B., Stingl, K., Gruntar, I., Ocepek, M., et al. (2015). High genetic similarity of ciprofloxacin-resistant Campylobacter jejuni in central Europe. Front. Microbiol. 6:1169. doi: 10.3389/fmicb.2015.01169

Lin, J., Akiba, M., Sahin, O., and Zhang, Q. (2005a). CmeR functions as a transcriptional repressor for the multidrug efflux pump CmeABC in Campylobacter jejuni. Antimicrob. Agents Chemother. 49, 1067-1075. doi: 10.1128/AAC.49.3.1067-1075.2005

Lin, J., Cagliero, C., Guo, B., Barton, Y. W., Maurel, M. C., Payot, S., et al. (2005b). Bile salts modulate expression of the CmeABC multidrug efflux pump in Campylobacter jejuni. J. Bacteriol. 187, 7417-7424. doi: 10.1128/JB.187.21.7417-7424.2005

Luangtongkum, T., Jeon, B., Han, J., Plummer, P., Logue, C. M., and Zhang, Q. (2009). Antibiotic resistance in Campylobacter: emergence, transmission and persistence. Future Microbiol. 4, 189-200. doi: 10.2217/17460913.4.2.189

Luangtongkum, T., Morishita, T. Y., Ison, A. J., Huang, S., McDermott, P. F., and Zhang, Q. (2006). Effect of conventional and organic production practices on the prevalence and antimicrobial resistance of Campylobacter spp. in poultry. Appl. Environ. Microbiol. 72, 3600-3607. doi: 10.1128/AEM.72.5.3600-3607.2006

Luangtonkum, T., Shen, Z., Seng, V. W., Sahin, O., Jeon, B., Liu, P., et al. (2012). Impaired fitness and transmission of macrolide-resistant Campylobacter jejuni in its natural host. Antimicrob. Agents Chemother. 56, 1300-1308. doi: 10.1128/AAC.05516-11

Mattheus, W., Botteldoorn, N., Heylen, K., Pochet, B., and Dierick, K. (2012). Trend analysis of antimicrobial resistance in Campylobacter jejuni and Campylobacter coli isolated from Belgian pork and poultry meat products using surveillance data of 2004-2009. Foodborne Pathog. Dis. 9, 465-472. doi: $10.1089 /$ fpd.2011.1042
Miller, W. G., On, S. L. W., Wang, G., Fontanoz, S., Lastovica, A. J., and Mandrell, R. E. (2005). Extended multilocus sequence typing system for Campylobacter coli, C. lari, C. upsaliensis, and C. helveticus. J. Clin. Microbiol. 43, 2315-2329. doi: 10.1128/JCM.43.5.2315-2329.2005

Niwa, H., Chuma, T., Okamoto, K., and Itoh, K. (2001). Rapid detection of mutations associated with resistance to erythromycin in Campylobacter jejuni/coli by PCR and line probe assay. Int. J. Antimicrob. Agents 18, 359-364. doi: 10.1016/S0924-8579(01)00425-3

Pascoe, B., Méric, G., Yahara, K., Wimalarathna, H., Murray, S., Hitchings, M. D., et al. (2017). Local genes for local bacteria: evidence of allopatry in the genomes of transatlantic Campylobacter populations. Mol. Ecol. 26, 4497-4508. doi: $10.1111 / \mathrm{mec} .14176$

Pérez-Boto, D., López-Portolés, J. A., Simón, C., Valdezate, S., and Echeita, M. A. (2010). Study of the molecular mechanisms involved in high-level macrolide resistance of Spanish Campylobacter jejuni and Campylobacter coli strains. J. Antimicrob. Chemother. 65, 2083-2088. doi: 10.1093/jac/dkq268

Rodrigo, S., Adesiyun, A., Asgarali, Z., and Swanston, W. (2007). Antimicrobial resistance of Campylobacter spp. isolated from broilers in small poultry processing operations in Trinidad. Food Control. 18, 321-325. doi: 10.1016/j.foodcont.2005.10.011

Vacher, S., Menard, A., Bernard, E., Santos, A., and Megraud, F. (2005). Detection of mutations associated with macrolide resistance in thermophilic Campylobacter spp. by real-time PCR. Microbial. Drug Resist. 11, 40-47. doi: $10.1089 / \mathrm{mdr} .2005 .11 .40$

Van Looveren, M., Daube, G., De Zutter, L., Dumont, J. M., Lammens, C., Wijdooghe, M., et al. (2001). Antimicrobial susceptibilities of Campylobacter strains isolated from food animals in Belgium. J. Antimicrob. Chemother. 48, 235-240. doi: 10.1093/jac/48.2.235

Vester, B., and Douthwaite, S. (2001). Macrolide resistance conferred by base substitutions in $23 \mathrm{~S}$ rRNA. Antimicrob. Agents Chemother. 45, 1-12. doi: 10.1128/AAC.45.1.1-12.2001

Wang, Y., Zhang, M., Deng, F., Shen, Z., Wu, C., Zhang, J., et al. (2014). Emergence of multidrug-resistant Campylobacter species isolates with a horizontally acquired rRNA methylase. Antimicrob. Agents Chemother. 58, 5405-5412. doi: 10.1128/AAC.03039-14

World Health Organization (2011). Critically Important Antimicrobials for Human Medicine, 3rd ev Edn. Geneva: World Health Organization

Wu, Z., Sippy, R., Sahin, O., Plummer, P., Vidal, A., Newell, D., et al. (2014). Genetic diversity and antimicrobial susceptibility of Campylobacter jejuni isolates associated with sheep abortion in the United States and Great Britain. J. Clin. Microbiol. 52, 1853-1861. doi: 10.1128/JCM.00355-14

Zhou, J., Zhang, M., Yang, W., Fang, Y., Wang, G., and Hou, F. (2016). A seventeenyear observation of the antimicrobial susceptibility of clinical Campylobacter jejuni and the molecular mechanisms of erythromycin-resistant isolates in Beijing, China. Int. J. Infect. Dis. 42, 28-33. doi: 10.1016/j.ijid.2015.11.005

Zirnstein, G., Li, Y., Swaminathan, B., and Angulo, F. (1999). Ciprofloxacin resistance in Campylobacter jejuni isolates: detection of gyrA resistance mutations by mismatch amplification mutation assay PCR and DNA sequence analysis. J. Clin. Microbiol. 37, 3276-3280.

Conflict of Interest Statement: The authors declare that the research was conducted in the absence of any commercial or financial relationships that could be construed as a potential conflict of interest.

Copyright (C) 2018 Elhadidy, Miller, Arguello, Álvarez-Ordóñez, Duarte, Dierick and Botteldoorn. This is an open-access article distributed under the terms of the Creative Commons Attribution License (CC BY). The use, distribution or reproduction in other forums is permitted, provided the original author(s) and the copyright owner are credited and that the original publication in this journal is cited, in accordance with accepted academic practice. No use, distribution or reproduction is permitted which does not comply with these terms. 